\title{
3DnST: A Framework Towards Understanding Children's Interaction with Tinkercad and Enhancing Spatial Thinking Skills
}

\author{
Srinjita Bhaduri \\ Quentin L. Biddy \\ Jeffrey B. Bush \\ Abhijit Suresh \\ Tamara Sumner \\ srinjita.bhaduri@colorado.edu \\ Quentin.Biddy@colorado.edu \\ Jeffrey.Bush@colorado.edu \\ Abhijit.Suresh@colorado.edu \\ sumner@colorado.edu \\ University of Colorado Boulder \\ Boulder, Colorado, USA
}

\begin{abstract}
With the proliferation of 3D printing technologies in schools and makerspaces, there is a need for teaching 3D modeling to students. Learning 3D modeling enhances spatial thinking skills, an essential skill for success in STEM. Creating 3D models requires students to have a deep understanding of 3D space, including rotating and scaling. In this study, we propose a framework developed through video-coding from analyzing screen recordings of middle-school students' usage of a 3D modeling tool - Tinkercad. The proposed framework focuses on identifying challenges students encounter during 3D modeling. These challenges include spatial thinking skills, working with the Tinkercad interface, and mental model formation. We authenticated the framework by collecting and analyzing data from a 3D printing unit in three middle schools. Our results and subsequent analysis can guide educators and researchers on how to use this framework to support students in having productive learning experiences with Computer-Aided Design tools.
\end{abstract}

\section{CCS CONCEPTS}

- Human-centered computing $\rightarrow$ Field studies; Usability testing.

\section{KEYWORDS}

Middle-School Students; 3D Printing; 3D Modeling; Spatial Thinking Skills; Maker Education; 3D Modeling Challenges; Framework; Tinkercad

Permission to make digital or hard copies of all or part of this work for personal or classroom use is granted without fee provided that copies are not made or distributed for profit or commercial advantage and that copies bear this notice and the full citation on the first page. Copyrights for components of this work owned by others than the author(s) must be honored. Abstracting with credit is permitted. To copy otherwise, or republish, to post on servers or to redistribute to lists, requires prior specific permission and/or a fee. Request permissions from permissions@acm.org.

IDC '21, fune 24-30, 2021, Athens, Greece

(c) 2021 Copyright held by the owner/author(s). Publication rights licensed to ACM. ACM ISBN 978-1-4503-8452-0/21/06 . \$ \$15.00

https://doi.org/10.1145/3459990.3460717

\section{ACM Reference Format:}

Srinjita Bhaduri, Quentin L. Biddy, Jeffrey B. Bush, Abhijit Suresh, and Tamara Sumner. 2021. 3DnST: A Framework Towards Understanding Children's Interaction with Tinkercad and Enhancing Spatial Thinking Skills. In Interaction Design and Children (IDC '21), June 24-30, 2021, Athens, Greece. ACM, New York, NY, USA, 11 pages. https://doi.org/10.1145/3459990.3460717

\section{INTRODUCTION}

The ability of 3D printing to create tangible objects from 3D (threedimensional) models created using Computer-Aided Design (CAD) software has caught the interest of both adults and children. Driven by open-source hardware platforms and international competition, 3D printers have rapidly transitioned from expensive professional tools into affordable mainstream consumer devices [43]. Consumerfocused 3D printers have resulted in the Maker Movement [10] leading to an upsurge in opportunities for children to explore these technologies $[42,57]$. The Maker Movement has made its way into mainstream education and coincident with it there is a rise in $\mathrm{K}-12$ teachers and educators including 3D printing and 3D modeling in-school or out-of-school [10]. While maker education involving $3 \mathrm{D}$ printing is gaining momentum, researchers are studying the interaction of children with these technologies [4, 6, 42]. Research suggests that tinkering and making by utilizing these technologies can be used in the classroom to support Science, Technology, Engineering, and Mathematics (STEM) learning [10, 24, 46]. Bliksten [10] suggests 'making' supports contextualized learning in STEM and has the potential for deep student engagement in projects that often have unknown complexities, with the power to engage students in rich interdisciplinary work. Although there is a growing body of research on the impact of makerspaces and maker technologies on children, there are fewer studies on ways to make these technologies child-friendly, i.e., more approachable and conceptually meaningful for them. Novices who are first-time users of these technologies, be it children or adults, often find them discouraging due to usability and learnability issues [15, 32, 36, 42, 50]. This research addresses the gap and focuses on identifying challenges students face when using 3D modeling tools and suggests ways to 
effectively teach 3D modeling and printing at the middle-school level (age 11-13).

3D modeling tools, like Tinkercad [17] and SketchUp [37], 3D focused instructional materials, and training programs are available to novice 3D modelers but learning 3D modeling can still be daunting and requires a serious time commitment to master [35]. Teaching 3D modeling to students is typically through a prescribed followalong structure, where a facilitator demonstrates how to create an example 3D model and participants follow along on individual computers [23]. This approach presents challenges for both the facilitator and the youth participating in it. Often this approach to facilitating 3D modeling curriculum leads to youth becoming bored or distracted [23], while others fall behind or become waylaid by usability and learnability issues with the user-interface of the CAD software [23, 33]. An important factor to effectively create 3D models is to have a deep understanding of 3D space or in other words to develop spatial thinking skills that can contribute to the ability to visualize 3D models from different perspectives [30]. Osborn and Agogino [48] (p. 75) define, spatial thinking as, “.. a mental process that involves thinking about relationships between three-dimensional (3D) objects." Spatial thinking plays an important role in preparing students for future STEM careers, such as engineering and architecture, which rely on students understanding relationships of 3D objects $[30,60]$. To improve a student's likelihood of pursuing a STEM career, the focus should be given to improving their spatial thinking skills $[16,61]$. Researchers Eisenberg and colleagues [31, 47], have studied how reconfigurable sheets and construction kits can engage children in spatial tasks and enhance their spatial thinking skills through simple paper folding activities. Another effective approach to train spatial thinking skills is to practice 3D modeling [46]. Not only do 3D modeling and 3D printing provide opportunities for rapid prototyping but they also open up a gamut of possibilities to facilitate the development of spatial thinking skills.

However, when it comes to effectively creating functional 3D models, a lack of good spatial understanding can lead to faulty 3D models (see Figure 1), resulting in 3D printing issues, like failed prints due to gaps in-between shapes and/or misalignments between shapes $[9,42]$. These issues contribute to teachers and facilitators not feeling comfortable and confident in teaching 3D printing and 3D modeling to children. After informal discussions with teachers from different school districts in a Midwestern state in the US, the researchers were able to determine that there are not enough high-quality student-centered resources readily available for teachers to utilize when teaching 3D modeling and 3D printing even with access to $3 \mathrm{D}$ printers in their classrooms. Not only is there a lack of teaching resources but the 3D modeling tools are not tailored to the needs of middle-school students. As Mike Eisenberg [24] (p. 13) stated "one might create (say) fabrication tools that simply allow children to select a pre-designed toy object from a website and print it out at home; this is a likely future scenario, but it's hard to argue that it greatly improves the lives of (at least materially well-off) children. In this author's view, tools of this sort simply feed into the larger cultural tide of thoughtless consumption." Hence, there is a need to consider alternative approaches to engaging middle-school students in 3D modeling where they create the 3D designs themselves, instead of simply downloading models created by another individual, that support ways to develop and enhance students' spatial thinking and provide novice 3D modelers with scaffolds to create, debug, and successfully print their 3D models.

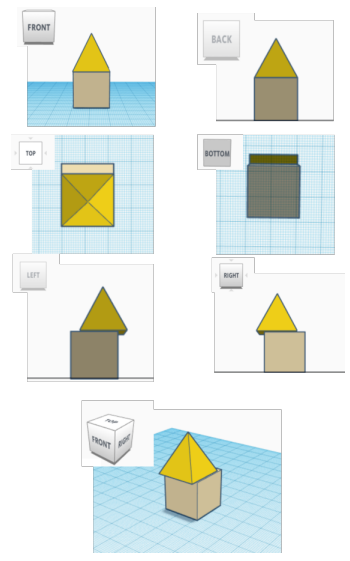

Figure 1: 3D model of a house that novices perceive as printable from FRONT and BACK view, but when rotated from other views like TOP, BOTTOM, LEFT, and RIGHT, novices realize that the shapes are not aligned.

The goal of this research is to answer the following research question, "What challenges do students encounter when creating $3 D$ models?". To do so, we video coded screen recordings of student interactions with a 3D modeling tool - Tinkercad. We conducted a pilot study in a large urban middle-school in a Midwestern state in the US in Spring 2020 and identified some common challenges students face, including issues with spatial thinking skills, working with the Tinkercad user interface, and student mental model formation. Next, to validate whether students concur with these identified challenges or "struggles", the research team conducted a series of studies in Fall 2020 in two schools in a small rural mountain community in the same Midwestern Mountain state. These schools are continuing this work. This paper focuses on analyzing and describing the experiences of students' interactions with and challenges encountered during creating their 3D models while engaging in an 8-week long 3D printing curriculum developed using an instructional design approach called storylining [52] to promote coherence, relevance, and meaning from the students' perspectives by using students' questions to drive the lessons. Students work on answering the driving question: "How can we support animals with physical disabilities so they can perform daily activities independently?". Our contributions include a framework describing and highlighting the 3D modeling challenges middle-school students can often encounter which we call "3D and Spatial Thinking” or 3DnST, with guidelines for teaching 3D modeling and 3D printing effectively to students by enhancing their spatial thinking skills.

\section{RELATED WORK}

Our work draws on prior studies of children's interaction with 3D printing and 3D modeling technologies, the benefits of learning 3D modeling, and ways it supports spatial thinking skills. 


\subsection{Opportunities of 3D Printing and 3D Modeling Technologies for Children}

3D printing is increasingly present in daily live especially in fields like engineering, science, architecture, healthcare, the food industry, and the fashion industry $[5,7,44]$. The creative and educational potential of 3D printing and 3D modeling makes it an excellent platform for children to design and print their 3D artifacts in schools, makerspaces, and libraries [10, 42, 57] and further develop their creativity, spatial thinking, and problem-solving skills. As Mike Eisenberg (2013) [24] envisioned almost a decade ago, 3D printing has become a prominent area of interest in education, it is becoming readily available to not only undergraduates and high-school students but also younger children [24]. Extensive research has followed the path that Eisenberg [24] (p. 8) put forth, "there is an acute need to consider how $3 D$ printing should develop, as a technology, to accommodate the abilities and activities of youngsters." 3D printing and 3D modeling technologies are becoming more childfriendly and there are several applications introducing youngsters to these technologies, both in-school and out-of-school. For example, 3D modeling tools like Tinkercad and Sketchup are used by students in elementary- and middle-school [35]. Low barrier 3D printers like Lulzbot and Makerbot are user-friendly and often used in elementary- and middle-schools or even as personal fabrication technologies at homes [55]. These technologies can support interdisciplinary work [10]. A study involving middle- and high-school teachers established that open-source 3D printing technologies have the potential to improve education by actively participating with these technologies and providing a sense of empowerment and cross-curriculum engagement [54].

The ability of 3D printers to create tangible artifacts from 3D designs generated digitally further supports student learning [41] and even has been beneficial to students with varied abilities like visual impairments $[13,58]$. In 2019, a literature review of several research articles [25] on the usage of 3D printing technologies in $\mathrm{K}-12$ education showed that around the globe there is a rise in engaging students in $3 \mathrm{D}$ printing. It can promote competencies such as computational thinking [25], design thinking [57], and has other intrinsic benefits of improving student self-esteem, collaboration skills, playfulness, and self-expression [10, 42]. Although there is a trend of encouraging children to engage in 3D printing there are several challenges: one of the first steps when $3 \mathrm{D}$ printing is designing a model within CAD software. Younger children have difficulty with the software's orientation, perspectives, floating shapes, and camera control $[6,9,42,50]$.

These difficulties when children design 3D models and issues with the CAD software provide opportunities to researchers to work towards solving these challenges. This work extends prior research by suggesting ways to maintain the engagement of children with these technologies by providing a framework identified through video-coding that recognizes challenges students encounter when using 3D modeling and 3D printing technologies. We put forward new approaches to identify and solve these challenges by studying ways to enhance children's spatial thinking skills through 3D modeling, a field that has been less studied and has enormous opportunities.

\subsection{Spatial Thinking Skills and 3D modeling}

Spatial thinking is the process of generating ideas through the spatial relationships between objects [48]. It is the form of mental activity that makes it possible to create spatial images and manipulate them in the course of solving practical and theoretical problems [62] (p. 55-56). Because space is a fundamental feature of the human environment, spatial thinking plays an important role in even the most ordinary human problem-solving. People process spatial information when they navigate, when they manipulate objects, and when they design them. All of us think spatially in everyday situations: when we consider rearranging the furniture in a room, when we assemble a bookcase, or when we relate a map to the road ahead of us [45]. Even to describe non-spatial situations we use spatial thinking, for example when we say one is close to a goal or describing someone as an outsider [28]. The cognitive processes involved in spatial thinking include feature decomposition, rotating, folding, orienting hypothesis generation, and verifying or checking hypotheses [29]. Researchers have studied the importance of spatial thinking in educational contexts and industry, with particular attention to STEM domains [51, 61, 64]. Longitudinal studies with high-school students show that spatial ability assessed during adolescence has contributed to being an important psychological attribute among those adolescents who eventually end up achieving success in STEM [34]. Results suggest that spatial ability plays a critical role in developing expertise in STEM and including spatial ability in modern talent searches could help identify adolescents who have these skills for STEM and are currently being deprived [64]. Others have investigated the relationship between spatial thinking ability and success in mathematics. Researchers have found that performance on spatial tests predicts success in mathematics and workplace problem solving [30].

Students who are trained and solve spatial thinking problems make better progress in improving their spatial thinking skills [30]. Researchers, Eisenberg and colleagues [31, 47] have studied how reconfigurable sheets and construction kits can engage children in spatial tasks and help enhance their spatial thinking skills through simple paper folding activities. Other research involving K-12 students created a mathematical 3D artifact, Wunderlich cube, comprising multiple spatial reasoning puzzles, has reported being beneficial in developing not only mathematical thinking but also computational thinking [20]. Recent research with students aged 11 to 15 have manifested that the process of 3D modeling helps develop spatial awareness skills [53]. These spatial abilities include understanding the perception of objects from different angles, mental construction and maintenance of visuals, and rotation and changing of shapes in the mind $[40,62]$. As mentioned earlier, several powerful 3D modeling tools or CAD systems are difficult for new learners to approach due to issues like confusing terminology and having to deal with complex geometries [23,33,38].

Even though powerful computers and software are available nowadays, developing visualization skills is necessary for imagining, specifying, and creating complex designs with functional features in the three spatial dimensions (X, Y, and Z). Students have difficulties in dealing with orthographic applications (projections, 
orthographic to isometric transformations, etc.) and perspectivechanging [38]. They seem to lack sufficient geometric and/or trigonometric relational skills, both of which are essential when modeling even simple geometric objects [66]. Tools like Autodesk's Tinkercad provide a lower barrier to entry with fewer unfamiliar words and simpler geometry [12, 35]. This, in turn, makes it convenient for children in K-12 to learn 3D modeling. However, 3D modeling tools often decompose the 3D designs into sequences of $2 \mathrm{D}$ interface operations [22]. 3D models created using modern CAD tools are not real 3D in some sense since they are isolated from the 3D physical world. Through this research, we identify challenges middle-school students face when creating 3D models and suggest ways teachers and educators, and CAD software developers, who are developing tools for children, can support features that make it intuitive for students to create 3D models effortlessly and find opportunities to develop their spatial thinking skills through the use of CAD without encountering common frustrations or being waylaid by difficulties they encounter.

\section{PILOT STUDY}

\subsection{Context}

In Spring 2020, before the Covid-19 pandemic, we conducted a pilot study in a large urban school in a Midwestern state in the United States. The study involved a 2-week implementation of the animal prosthetics curriculum. Students engaged in the engineering design process by defining, developing, and optimizing solutions to create and print prosthetic limbs for animals with disabilities using 3D modeling - Tinkercad, and 3D printing. Students used stuffed animals to use as reference for measurements (see Figure $2 b)$. Based on the recommendation from the district's STEM coordinator and the teacher's availability to implement a 3D printing unit, we conducted this pilot in a technology classroom consisting of $n=21$ ( 5 boys, 16 girls) in 7th and 8th grade. The teacher had prior experience teaching 3D printing and had access to the Flashforge $3 \mathrm{D}$ printer. Students reported having little to no prior experience in 3D modeling and 3D printing.

\subsection{Data Collection}

We collected data in the form of surveys, field notes, screen recordings of student interactions with Tinkercad, and student artifacts. In this article, we focus on the screen recordings of student interactions with Tinkercad to identify the challenges students face when creating 3D models. Over the 9 days (2-weeks) of the implementation, each 40-minute class period was video-taped with cameras focused on selected student computer screens to capture their interactions with the Tinkercad workspace. Students whose interactions were videotaped self-reported themselves as novices with no experience using Tinkercad but minimal experience with the MakersEmpire CAD interface. Our goal was to better understand what novices or first-time Tinkercad users struggle with when creating 3D models. In the next section, we describe our approach to analyze these videos, the tasks students performed using Tinkercad, our approach to creating a codebook for coding the screen recordings for 3D modeling challenges, and finally present the 3DnST framework.

\subsection{Data Analysis}

To analyze the data collected from the pilot study we first conducted an initial round of data reduction [39], where we looked at only videos for students who provided consent and watched each of the 40 minutes looking for student interactions with Tinkercad which involved students creating their unique 3D model. Our analysis includes a corpus of three 40-minute videos of one student's [P1] interaction with Tinkercad. The other videos were excluded from this analysis since in these videos students were only working on Tinkercad tutorials, learning the 3D printing process, had students researching animal prosthetics, or were not as rich and informative as P1's interaction with Tinkercad.

We then analyzed the selected videos to develop a coding framework that includes a list of organizational codes and subcodes that identifies minuscule challenges students may face. In this article, we use examples from P1's Tinkercad interactions to show how our coding framework was developed and the ways this framework identifies common challenges researchers, teachers, and educators often find their students struggling with when creating 3D models. Creating qualitative coding frameworks often depends on a balance between etic/outsider and emic/insider knowledge, decisions between coders on interpretation, and practical compromises about labels and meanings [1]. To maintain this balance our video-coding team included a mix of three researchers with varied experiences with 3D modeling. The first author had prior experience teaching 3D modeling in K-12 and two other researchers on the team had experience with $\mathrm{CAD}$ but had much less experience teaching 3D modeling.

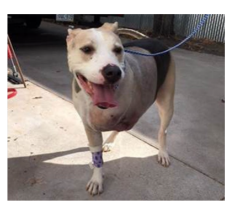

(a)

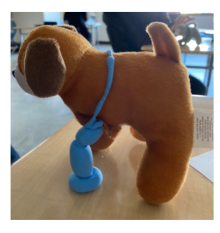

(b)
Figure 2: (a) Nalla, a dog with prosthetic needs that students were creating a 3D model prosthetic for, (b) Stuffed animal that looks like Nalla with an amputated left front leg for students to use as reference for measurements

\subsection{Video coding procedure}

Our team conducted multiple rounds of coding and analysis, starting with an initial deductive analysis followed by an inductive analysis to generate themes [19]. Existing theories informed these themes we had about challenges students face when creating 3D models i.e., software's orientation, perspectives, floating shapes, alignment issues, and camera control $[6,42,50]$. We identified similar challenges in our previous work with high-school students when they were creating 3D models of chess pieces and drone attachments $[8,9]$. In the selected video segments for this paper, students were creating 3D prosthetic for Nalla, a three-legged dog missing a front leg (see Figure 2a and Figure 2b). 
Overall, our deductive analysis involved an attempt to categorize all possible challenges identified in the videos including on-task time, and off-task time. We use on-task and off-task time as the primary category since prior research suggests that there is a positive relationship between on-task time and achievement, although other factors play a role in this measurement of time [27]. We included some of these other factors in our measurement of on-task time, like seeking help, or working on other task related activities. The unit of analysis for this coding system was every 30 second interaction students had with Tinkercad, focusing on when the primary student [P1] is on-task working directly on their 3D project and if so, identifying whether the student is working independently or seeking help and next identifying any challenges the student encountered. In our first round, all researchers coded one 40 minute video (V1), after discussing disagreements, the codebook was revised and used for the next phase of analysis. In the second round, we coded a different video (V2) and in our final pass, we double coded the first video (V1) using our final agreed on codebook. Since the 40-minute video consisted of multiple segments where the student was off-task, we decided to double code the entire 40 minutes of V1. Among the three coders, we reached a $65 \%-80 \%$ reliability by calculating Cohen's Kappa [65]. Once a Kappa of substantial agreement was reached, the first author coded the final video V3.

In the first round of coding with V1, we began by independently marking the beginning and end of segments determined to be ontask. However, in independently determining these segments each researcher identified segments for different lengths of time and/or for what were the primary challenges students encountered. In the second-round with V2, we decided to divide each unit of analysis into 30 seconds in order to synchronize our noticings and make them more consistent as well as to capture a more fine grained analysis of the challenges students encountered. Some of the segments were adjusted by a few seconds as necessary to better capture an interaction. When coding we followed certain rules to determine on-task and off-task time and in identifying each challenge. The on-task time of the primary student was coded as working independently with Tinkercad or seeking help with creating their 3D model. Once it was determined that the student was either working independently or seeking help we noted what the student was doing and what challenges they were encountering (including a code for "no challenge" if they were working independently without any issues). We coded for up to six different challenge categories (0-5) (see Figure 3). If we were unable to determine from the audio what a student was saying but were able to discern from the screen recording if they were pointing to their screen, or if a teacher/more knowledgeable others revoice confirm it, we coded the clip appropriately. Moreover, if the observed primary student was not "driving" the screen, if someone else completely took over editing or creating the design for them, we coded it as off-task. We utilized the resulting coding framework to double code V1.

Additionally, we created an exemplar reference video showing one ideal process for creating the prosthetic leg, we referred to this video when there was a need for comparing student screen recordings to an ideal scenario of approaching the $3 \mathrm{D}$ model design. Through inductive analysis, we identified some of the issues students face when trying to create a design they have in mind i.e, their mental model formation of the designs [14] vs the objects and shapes available to them on Tinkercad. Analysis of the videos is presented as the 3DnST framework in the next section.

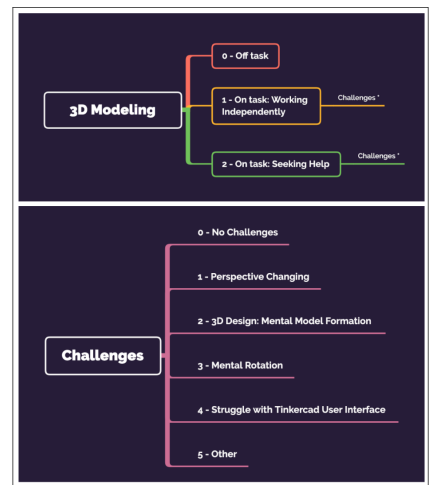

Figure 3: Overview of 3DnST Framework and the 3D modeling interaction coding manual

\section{3D AND SPATIAL THINKING (3DNST) FRAMEWORK}

Our goal behind developing the 3DnST framework was to provide a common language to use for identifying and addressing 3D modeling challenges students commonly face, behaviors identified when children work on 3D modeling tasks and a foundation on which research can build [18]. Introducing 3D printing in schools and makerspaces, and going over built-in tutorials on Tinkercad is not enough for students to learn these technologies. There is a current need in this area to define best practices and better understand how to utilize these technologies for the purpose of effective learning regarding 3D modeling, maintaining student engagement and interest with these technologies, and supporting spatial thinking development. In the following section, we identify the central challenges students face when creating 3D models. This list of challenges is by no means meant to be exhaustive but instead is offered here as a foundation on which to build and expand through further research.

\subsection{D Modeling Task}

The 3DnST framework identifies three categories of activities (see Figure 3) performed by students in an in-class setting and working on a 3D modeling task on a CAD software platform. We focused on 3D solid modeling using a web-based 3D tool - Tinkercad. The three high-level classifications of the activities involve:

- Off-task: Off-task behavior includes off-task peer interactions, self-distraction, and off-task behaviors directed towards aspects of the classroom environment $[3,26]$. If a student's attention seems distracted and their behavior does not include them working on Tinkercad or completing the task they have been assigned then we identify it as off-task. This might include being distracted looking at emails, checking other materials on the internet, talking to their peers about topic not related to the task of creating the prosthetic leg, or at any point if we find them not in their seat interacting with the 3D modeling workspace. Exceptions: if they are using 
the internet to search for something related to their struggle with the Tinkercad user interface then it does not fall under this off-task category or if we find them researching about the animals they are working on or prosthetic design.

- On-task, Working Independently: On-task behavior on the other hand [3,26] includes students actively working on their 3D models. For example, they interact with different shapes to create a 3D design. In this category, we recognized students actively working on their computers, independently, to create their 3D design using Tinkercad and not engaged in off-task behaviors described above.

- On-task, Seeking Help: Due to the challenges that come with learning $\mathrm{CAD}$, there is a pattern among novices in seeking help from a more knowledgeable other (AKO), be it their peer, teacher, or another adult [63]. When seeking help, novices tend to struggle with formulating questions, conveying the visual context, and geometry-specific conversations [15]. Observed behaviors for on-task seeking help include students discussing or brainstorming with another person about their 3D model. Students in classrooms co-located with AKO tend to ask questions related to the 3D design, the Tinkercad user interface, or any other challenges when creating the 3D model.

\subsection{D Modeling Challenges}

The identified challenges presented in this framework are based on prior literature, the video coding analysis conducted from the pilot study data, and the first author's prior field experience. Of the six identified types of challenge (see Figure 3), four are described below and can be categorized under two overarching categories of 1) spatial thinking skills: perspective-changing, 3D mental model formation, and mental rotation, and 2) Tinkercad interface. The other two challenge types are briefly described here. The "no challenge" code was selected when the student was working independently but not encountering any challenges. The "other" code was selected when the student faced challenges that did not fit clearly into the other challenge codes. Based on our analysis, we theorize that the commonly observed challenges do indeed belong in these two overarching categories. We also posit that using this framework might provide ways to scaffold 3D instruction to train and help students improve these skills. Additionally, this framework can be taken into consideration when designing CAD tools for children with varied abilities.

4.2.1 Perspective-Changing. One of the spatial thinking skill categories is perspective-changing/taking. Perspective changing is the ability to imagine how an object or scene looks from different perspectives to another observer [38]. Often students struggle with different views or perspectives when creating 3D models. They do not instinctively rotate to different perspectives to create or debug their models. In not doing so, they are not viewing the model from different angles and working in only one view/perspective. This can result in students having floating shapes on the Tinkercad Workplane (see Figure 4, View 2), have gaps in their 3D models (see Figure 4, View 3), and/or misaligned shapes (see Figure 4, View 2) [42]. One strategy we often see students employ to solve this issue is zooming in and out to create the design and identify any errors their designs might have. This is not an ideal strategy for debugging their 3D models, hence we suggest alternate approaches to make perspective changing more intuitive for novice students. On the other hand, in Figure 4 View 1, snippets from the video/screen recordings we analyzed, we noticed that the student added shapes from that particular view and right after that the neighbor rotated the screen and they could see the floating shape (cylinder), as in Figure 4, View 2.

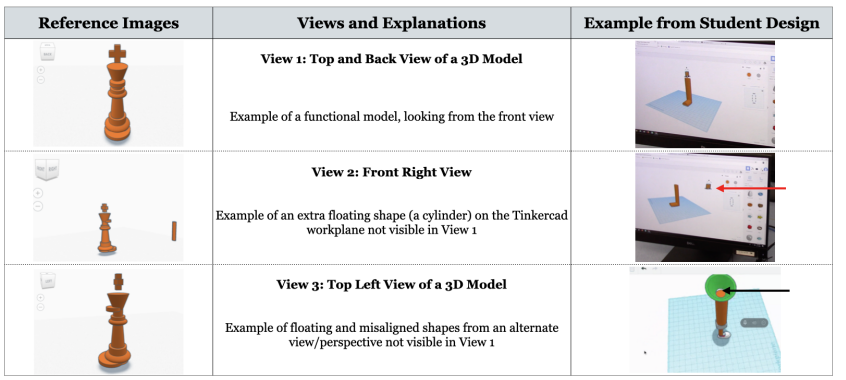

Figure 4: Examples of different views of 3D models in Tinkercad and possible issues that could arise. On the left column are images of reference issues using a chess piece, the center column shows the different views and explanations, and the right column contains examples of student designs we noticed in the pilot study videos and the challenges they encounter.

4.2.2 3D Design: Mental Model Formation. Another spatial thinking category, mental model formation, is the ability to piece objects together to create more complex shapes or visualizations and the ability to mentally transform objects, often from a $2 \mathrm{D}$ sketch to 3D design or vice versa [45]. During creating 3D models from 2D sketches and when selecting built-in 3D shapes on Tinkercad, students can have difficulty understanding which shapes to choose, how the representation of their sketch will look like in 3D on Tinkercad, issues with accurate measurements and scaling of shapes to match the object they are creating, and proportions of objects concerning other shapes/objects. For example, when students are creating 2D sketches of their prosthetic design many are unable to understand what shapes to use to create a $3 \mathrm{D}$ version. On the contrary, when translating from $3 \mathrm{D}$ to $2 \mathrm{D}$, students have difficulty relating their $2 \mathrm{D}$ sketch to the model they made.

4.2.3 Mental Rotation. Mental rotation, another challenge with spatial thinking skills is the mental ability to rotate either $2 \mathrm{D}$ or $3 \mathrm{D}$ objects to a certain angle in either clockwise or anticlockwise direction $[11,45]$. Students often are not able to mentally rotate objects and need to either physically orient their head in order to figure out how to mentally rotate objects in their model. In Tinkercad, students often struggle to understand how to create a particular design and the degree of rotation needed for specific shapes. In the king chess piece example (see Figure 5), the cross on top involves the need for mental rotation. Students must consider how much the orange boxes should be rotated and which direction (clockwise/anticlockwise) they should be rotated in order to create the cross shape. 


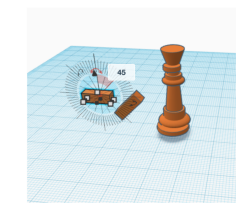

Figure 5: An example of mental rotation challenges

4.2.4 Tinkercad User Interface. Tinkercad as a CAD tool has a low barrier to entry for students but even though the user interface has been made child-friendly, interacting with it is often demanding for students [35]. Students usually struggle to know how to group shapes to create a new complex shape, are unable to move shapes around on the Tinkercad Workplane, are unsure how to increase the height of objects/shapes, and have difficulty resizing objects. These were common challenges identified in our screen recordings. The 3DnST framework was developed for educators, researchers, teachers, and CAD developers to build on to provide a richer and more user-friendly CAD environment in which students can more easily create 3D models with minimal frustration and challenges. In order to authenticate these findings related to the 3DnST framework we subsequently surveyed a larger audience of students to identify if they encounter similar challenges. In the following section, we elaborate on this approach and findings.

\section{3D CHALLENGES SURVEY STUDY: METHODS}

\subsection{Context}

In Fall 2020, we studied n=397, 7th and 8th graders, from two different schools in a rural mountain school district. These students were predominantly Hispanic (70\%), economically disadvantaged (60\% qualify for free and reduced lunch); and English Language Learners (ELL) (67\%). As per the school district's Covid-19 pandemic regulations, teachers taught four days in-person with some students opting to join remotely, and one day for asynchronous learning and teacher lesson planning. In-person days consisted of five, one-hour long class periods. Ideally, these classrooms would be studied in-person but given the pandemic, all interactions between the researchers and the teachers and their classrooms had to be remote and virtual. We had to explore new ways to provide curriculum resources and professional development, develop new research instruments along with methods to observe and collect data from a complete remote context. The same 3D printing curriculum utilized in the pilot study was implemented in this survey study but this context allowed for a longer and much richer implementation including more hands-on activities (albeit socially distanced), and increased opportunities for students to iterate on their 3D animal prosthetic designs. Each student was provided a stuffed animal that our research team had stitched and altered so that one of the legs, randomly selected (i.e., front, rear, right, or left) were amputated in order to give students a physical, scaled model of an animal to use when creating their 3D models (see Figure 2b). All the curriculum tasks and tools were kept similar in order to ensure consistency between the pilot study and the survey study.

\subsection{Data collection and instruments}

During the 3D challenges survey study, investigating the 3DnST framework for challenges, data was collected from a student initial interest survey on 3D printing, Tinkercad experience surveys, and post-implementation semi-structured student interviews. Interviews elicited students' perceptions of their overall experience with the curriculum, their understanding of Tinkercad and 3D modeling, in addition to other formative feedback. Interviews lasted approximately 15 minutes, were conducted via video conference, and were automatically transcribed by the video conferencing tool. We interviewed 34 students selected by the teachers from each of their class periods based on which students had informed parent consent and student assent and which students were willing to be interviewed by the research team.

The pre-implementation interest survey was administered at the beginning of the curriculum and involved students self-reporting their experience with 3D printing and 3D modeling, and any prior $\mathrm{CAD}$ tools used. All surveys were translated into Spanish to support the large ELL population and all surveys were administered digitally through Google forms. The Tinkercad experience survey included both multiple-choice and open-ended questions and utilized the challenges identified in the 3DnST framework posing the challenges as multiple-choice questions. This survey was one way the research team adapted protocols from the pilot study to support the inability to study classrooms in-person due to the Covid-19 pandemic. Research personnel were not permitted in classrooms to do video-recordings of students interacting with Tinkercad and the curriculum similar to the pilot study, so administering the surveys was an ideal way to collect relevant comparable data. The Tinkercad survey was administered in the 6th-week of the curriculum after students had at least created the initial prototype of their prosthetic devices.

\subsection{Data Analysis}

Data collected from surveys were cleaned and pre-processed to ensure there were no missing responses. The first survey administered, the pre-implementation initial interest survey, 303 of the total 397 students completed this survey. We began the analysis by grouping different students based on their self-report of 3D modeling experience ratings on a scale of $1-5$, where 1 : Novice - no experience with 3D modeling; and 5: Expert - a lot of experience with 3D modeling. Similarly, the Tinkercad experience survey was cleaned and pre-processed based on student identifiers from the initial interest survey. Out of the 303 students who responded to the initial survey, only 163 students responded to both the initial interest and Tinkercad experience surveys. In addition to the surveys, the transcribed interviews were analyzed to identify student responses consistent with our proposed list of 3D printing and modeling challenges. We also sought evidence for student quotes that would inform and add to our knowledge of common challenges that students encountered when working with Tinkercad.

\section{FINDINGS}

Our findings focus on the challenges students reported when 3D modeling and the help they needed for creating their 3D designs, in order to compare it with our proposed 3DnST framework. 


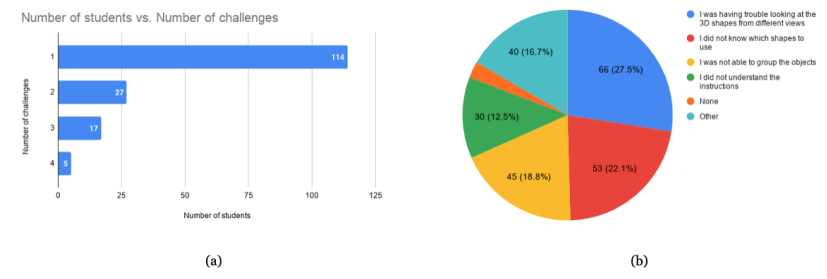

Figure 6: (a) Bar plot showing the distribution of challenges vs students i.e., how many students (out of 163) faced one or more challenges, (b) pie chart illustrating the distribution of 240 student responses to $3 \mathrm{D}$ modeling challenges.

\subsection{Challenges with 3D modeling}

Out of the 163 students who completed both the initial interest and Tinkercad surveys, 49 students responded saying they faced more than one challenge and 114 students said they faced at least one challenge (see Figure 6a). To simplify the data collected from the 49 students who faced more than one challenge, we divided up the responses into individual challenges. In total, we collected $\mathbf{2 4 0}$ individual responses across the 163 students who completed both the initial interest and Tinkercad surveys (see Figure 6b). As in Figure 6 b, with $27.5 \%$ (66) of the individual responses showed students having difficulty with looking at their 3D model from the different views, perspective changing was the most common challenge that the students encountered. Similarly, 22.1\% (53) of student responses showed they struggled with selecting the shapes (3D design: mental model formation) for creating their prosthetic leg. Further, a total of $18.8 \%$ (45) of the student responses showed they had trouble grouping shapes together (Tinkercad user interface). Also, there was a small portion of students i.e., 12.5\% (30) who reported having difficulty with the instructions, and 6 students who said they did not face any challenge at all and were comfortable with using Tinkercad. Finally, 16.7\% (40) of the responses included "Other" as an option where students could select other challenges they encountered that were to some extent a rewording of the four identified challenges. For example, in the surveys, students reflected on their inability for perspective changing saying "I could not get the shape in the places I wanted it at times and that made it a little difficult." While other students reflected on facing challenges related to their experience as a novice using Tinkercad, "Making things not stick together so they can move later" or the 3D mental model formation where a student reflected "It's kind of hard to create what you make in your mind then create it." There were responses that indicated students felt they can get better with 3D modeling and Tinkercad, but need more persistence in the 3D modeling process, "it wasn't hard it just took up time".

Responses from the post-implementation semi-structured interviews support our findings from the surveys. When asked "What was challenging in Tinkercad?" students had varied responses as seen in Table 1. The common challenges include perspective changing, Tinkercad user interface, and student mental model formation of the 3D designs.

Table 1: Student interview responses (quotes) when asked about their experiences with 3D modeling and Tinkercad and the corresponding challenges tied to the 3DnST framework, the challenges are mentioned in the order they appear in the quotes

\section{Students Responses first of all, as I said earlier, get- ting the measurements right with the measurement of the ani- mal and also like combining all these different shapes to make one, making sure there are no holes in anything. And then you have to like, look around the whole thing. Make sure it's all the size you want."}

The measurement was the hardest part, especially the gaps. Tinkercad is hard to use.

Putting the pieces together and moving them, making them bigger, smaller.

Like it's kind of hard to navi- Tinkercad user interface gate. But once I get it makes more Controls sense.

\subsection{Help-seeking for novice students}

As described in our findings for the common challenges, we had similar responses for students who sought help. Of the 163 responses from the Tinkercad experience survey $82.20 \%$ (134) of students reported having faced at least one of the four challenges. While $47.85 \%$ (78) of students reported they needed help when creating their 3D models, a total of $43.5 \%$ (71) students sought help and also faced challenges. Most students sought help from a classmate, teacher, or searched online. The common challenges students needed help included the ability to group shapes, rotation of shapes and objects, measurements, and determining which shapes to use. In Table 2, we provide a break-down of the student's self-reported 3D modeling ability in relation to the corresponding number of students who struggled with Tinkercad and sought help when creating the 3D model of the prosthetic leg.

\section{DISCUSSION}

Our goal in conducting this work has been to explore challenges students encounter when creating 3D models using Tinkercad and ways these challenges can be categorized to create and support child-friendly interactions with CAD. Preliminary results provide evidence for the potentiality of this framework to identify the common challenges faced by students during 3D modeling. Leveraging 
Table 2: Summary of responses from the Initial Interest and Tinkercad Experience Surveys

\begin{tabular}{|c|c|c|c|c|}
\hline Likert Scale Rating & $\begin{array}{l}\text { 3D modeling skill level: } \\
\text { total responses from } \\
\text { Tinkercad experience } \\
\text { and initial interest } \\
\text { surveys }\end{array}$ & $\begin{array}{l}\text { Total students who re- } \\
\text { ported at least one of } \\
\text { four challenges }\end{array}$ & $\begin{array}{l}\text { Total students who } \\
\text { sought help }\end{array}$ & $\begin{array}{l}\text { Total students who } \\
\text { faced challenges and } \\
\text { sought help }\end{array}$ \\
\hline 1 - Novice & 51 & 45 & 26 & 25 \\
\hline 2 & 48 & 38 & 22 & 18 \\
\hline 3 & 47 & 40 & 25 & 23 \\
\hline 4 & 14 & 10 & 5 & 5 \\
\hline 5 - Expert & 3 & 1 & 0 & 0 \\
\hline Total & 163 & 134 & 78 & 71 \\
\hline
\end{tabular}

results from the current and prior studies [6, 9, 42, 50], the 3DnST framework is able to outline two high-level categories of challenges in terms of spatial thinking skills and Tinkercad user interface issues: 1) spatial thinking skills: perspective-changing, 3D mental model formation, and mental rotation, and 2) Tinkercad interface. We collected both qualitative and quantitative data from screen recordings, semi-structured interviews, and surveys in order to generate the 3DnST framework and our findings from the analysis illustrate evidence for correspondence between seeking help, facing challenges, and student 3D modeling experience level. Novice students (experience level 1 - 3) are likely to face more challenges and seek guidance while students who consider themselves as experts (experience level 4, or 5) do not encounter as many challenges and figure out any challenges themselves rather than seeking help. This proposed work clearly reiterates the need for creating a more user-friendly platform for children. Our goal as educators is to encourage more students to experience the benefits of CAD and rapid prototyping instead of getting frustrated or waylaid by the learnability and usability issues [23, 33].

We realized most students struggle with the understanding of 3D space even though they are exposed to video games and other technologies $[45,61,62]$ that enhance spatial thinking skills. These struggles are often the reason why students are uncomfortable using 3D printing and 3D modeling technologies. In our video analysis from the pilot study, we observed the student constantly zooming in and out to align the shapes even after being shown how to rotate the Tinkercad workplane to view it from other perspectives. Even with persistent efforts, some students were unable to fix gaps in their models and ultimately had to seek help from peers to fix the problem. Similarly, from discussions with the teachers, we identified that students who are unable to identify gaps in their 3D designs often had a 3D print failure or "spaghetti print". As seen in Figure 4 View 3, the student was unable to identify the gap until the teacher pointed it out. Such gaps and misalignments are reasons for 3D print failures and lead to frustrations for students that can be a barrier to student engagement with 3D modeling and printing. Students need to have a deep understanding of the 3D space (i.e., spatial thinking skills) or have an opportunity to debug their 3D models before 3D printing them.

This framework is novel in that it can serve to provide guidelines for teachers to develop questions and strategies with a student centered focus that can help students figure out how to move past challenges they encounter with $3 \mathrm{D}$ printing and modeling. Utilized as a set of guidelines, teachers could use this framework to anticipate and prepare for the challenges students are most likely to encounter and make plans for how to support them in problem solving through these challenges. We believe if students are given the opportunity to iterate on their designs, over time making multiple sketches of their designs and then creating 3D models, we can allow for better development of 3D mental models. It can support the development of their spatial thinking skills around 2D to 3D translation and vice-versa. Finally, an approach to maintain student engagement with 3D printing and 3D modeling technologies is to use a curriculum that is relevant and coherent from the students perspective [49,56], like in our curriculum students were realizing the need to solve a real-world problem of helping animals in need. Our work surrounding the 3DnST framework has determined that opportunities exist for supporting and enhancing spatial thinking skills in middle-school students and we envision these opportunities extending to both elementary- and high-school and other novice adult 3D modelers using CAD technologies in their daily life or career.

\section{FUTURE WORK}

We present the 3DnST framework and the 3D modeling challenges to educators and researchers and suggest using scaffolds when teaching 3D modeling to students. Children are not able to touch or grasp virtual models. To address this problem, researchers are focusing on separating graphics out of conventional displays and integrating them into the physical world. One such scaffold is using Augmented Reality, prior work has shown that AR can support the $3 \mathrm{D}$ modeling process [21, 59]. Augmented Reality (AR) [2] technology could provide a solution to this problem. The potential benefits of AR are improvements in the students' abilities with respect to spatial cognition, concept development, decision making as well as design modifications and refinements due to the support for viewing and touching the design. Considering these benefits AR offers, our team is working towards field-testing an easy-to-use childfriendly AR app we developed to support debugging of 3D models before $3 \mathrm{D}$ printing. We believe similar emerging technologies can be integrated to allow real-world visualization of 3D models. 
While, our work has looked at one students' interaction with Tinkercad to begin to develop the 3DnST framework. Through our approach to test and generalize our framework to a larger population in our second study we have developed a platform that students agree with and we have been able to successfully use it to study children's interactions with $\mathrm{CAD}$ and further improve our framework to include other challenges. In the future, in a non-pandemic world where we will be present in classrooms to deeply engage with children and develop richer technologies for the future generation of children we can further test and expand this framework to be more comprehensive and applicable to students in other demographics and age ranges.

\section{SELECTION AND PARTICIPATION OF CHILDREN}

We collaborated with three STEM and technology teachers teaching 7th and 8th grade students at two different middle school districts in a Midwestern state in the US. Students in the pilot study received an explanation about our research, and were assured that any faces would be blurred from images that might be utilized in research articles or publications. We video-taped only students who wished to participate and provided consent to be videotaped. For the 3D Challenges survey study, we provided digital consent forms to teachers and they distributed physical copies in their classrooms. In their class-periods, teachers explained the form and the research study to students. Students who consented were interviewed and compensated with a 5 gift card. All identifiable student information was anonymized.

\section{ACKNOWLEDGMENTS}

We thank the anonymous reviewers for their valuable suggestions and feedback. This material is based upon work supported by the National Science Foundation under Grants No. 1948709 and 1742053. We thank our collaborators on these grants for their constant feedback and support. This work would not have been possible without the teachers, students, and administrators of the participating school districts for their patience with data collection and supporting this research, even during the pandemic.

\section{REFERENCES}

[1] Jennifer Keys Adair and Giulia Pastori. 2011. Developing qualitative coding frameworks for educational research: immigration, education and the Children Crossing Borders project. International fournal of Research \& Method in Education 34, 1 (2011), 31-47.

[2] Ronald T Azuma. 1997. A survey of augmented reality. Presence: Teleoperators \& Virtual Environments 6, 4 (1997), 355-385.

[3] Ryan SJd Baker. 2007. Modeling and understanding students' off-task behavior in intelligent tutoring systems. In Proceedings of the SIGCHI conference on Human factors in computing systems. 1059-1068.

[4] David Bar-El and Marcelo Worsley. 2019. Tinkering with Music: Designing a Maker Curriculum for an After School Youth Club. In Proceedings of the 18th ACM International Conference on Interaction Design and Children. 220-226.

[5] Patrick Baudisch and Stefanie Mueller. 2016. Personal fabrication: State of the art and future research. In Proceedings of the 2016 CHI Conference Extended Abstracts on Human Factors in Computing Systems. 936-939.

[6] Alexander Berman, Elizabeth Deuermeyer, Beth Nam, Sharon Lynn Chu, and Francis Quek. 2018. Exploring the 3D printing process for young children in curriculum-aligned making in the classroom. In Proceedings of the 17th ACM Conference on Interaction Design and Children. 681-686.

[7] Srinjita Bhaduri, Jesús G Ortiz Tovar, and Shaun K Kane. 2017. Fabrication Games: Using 3D Printers to Explore New Interactions for Tabletop Games. In Proceedings of the 2017 ACM SIGCHI Conference on Creativity and Cognition. 51-62.
[8] Srinjita Bhaduri, Katie Van Horne, John Daniel Ristvey Jr, Randy Russell, and Tamara Sumner. 2018. From Toys to Tools: UAVs in Middle-school Engineering Education (RTP). In 2018 ASEE Annual Conference \& Exposition. ASEE Conferences, Salt Lake City, Utah. https://peer. asee. org/30546.

[9] Srinjita Bhaduri, Katie Van Horne, and Tamara Sumner. 2019. Designing an Informal Learning Curriculum to Develop 3D Modeling Knowledge and Improve Spatial Thinking Skills. In Extended Abstracts of the 2019 CHI Conference on Human Factors in Computing Systems. 1-8.

[10] Paulo Blikstein. 2013. Digital fabrication and 'making' in education: The democratization of invention. FabLabs: Of machines, makers and inventors 4 (2013), $1-21$.

[11] Catherine D Bruce and Zachary Hawes. 2015. The role of 2D and 3D mental rotation in mathematics for young children: what is it? Why does it matter? And what can we do about it? ZDM 47, 3 (2015), 331-343.

[12] Erin Buehler, William Easley, Samantha McDonald, Niara Comrie, and Amy Hurst. 2015. Inclusion and education: 3D printing for integrated classrooms. In Proceedings of the 17th International ACM SIGACCESS Conference on Computers \& Accessibility. ACM, 281-290.

[13] Erin Buehler, Shaun K. Kane, and Amy Hurst. 2014. ABC and 3D: Opportunities and Obstacles to 3D Printing in Special Education Environments. In Proceedings of the 16th International ACM SIGACCESS Conference on Computers \& Accessibility (Rochester, New York, USA) (ASSETS '14). Association for Computing Machinery, New York, NY, USA, 107-114. https://doi.org/10.1145/2661334.2661365

[14] Kirsten R Butcher. 2006. Learning from text with diagrams: Promoting mental model development and inference generation. Journal of educational psychology 98, 1 (2006), 182.

[15] Parmit K Chilana, Nathaniel Hudson, Srinjita Bhaduri, Prashant Shashikumar, and Shaun Kane. 2018. Supporting remote real-time expert help: Opportunities and challenges for novice 3d modelers. In 2018 IEEE Symposium on Visual Languages and Human-Centric Computing (VL/HCC). IEEE, 157-166.

[16] Douglas H Clements. 2004. Geometric and spatial thinking in early childhood education. Engaging young children in mathematics: Standards for early childhood mathematics education (2004), 267-297.

[17] Lydia Cline. 2014. 3D Printing with Autodesk 123D, Tinkercad, and MakerBot. McGraw-Hill.

[18] Jonathan Cohen, W Monty Jones, Shaunna Smith, and Brendan Calandra. 2017. Makification: Towards a framework for leveraging the maker movement in formal education. Fournal of Educational Multimedia and Hypermedia 26, 3 (2017), 217-229.

[19] John W Creswell and Cheryl N Poth. 2016. Qualitative inquiry and research design: Choosing among five approaches. Sage publications.

[20] R Devon, RS Engel, RJ Foster, D Sathianathan, and GF Turner. 1994. The effect of solid modeling software on 3-D visualization skills. Engineering design graphics journal 58, 2 (1994), 4-11.

[21] Trien V Do and Jong-Weon Lee. 2009. A multiple-level 3D-LEGO game in augmented reality for improving spatial ability. In International Conference on Human-Computer Interaction. Springer, 296-303.

[22] Trien V Do and Jong-Weon Lee. 2010. 3darmodeler: a 3d modeling system in augmented reality environment. International fournal of Electrical, Computer, and Systems Engineering 4, 2 (2010), 145-154.

[23] Volodymyr Dziubak, Ben Lafreniere, Tovi Grossman, Andrea Bunt, and George Fitzmaurice. 2018. Maestro: Designing a System for Real-Time Orchestration of 3D Modeling Workshops. In Proceedings of the 31st Annual ACM Symposium on User Interface Software and Technology. 287-298.

[24] Michael Eisenberg. 2013. 3D printing for children: What to build next? International fournal of Child-Computer Interaction 1, 1 (2013), 7-13.

[25] Simon Ford and Tim Minshall. 2019. Invited review article: Where and how 3D printing is used in teaching and education. Additive Manufacturing 25 (2019), $131-150$

[26] Karrie E Godwin, Ma V Almeda, Howard Seltman, Shimin Kai, Mandi D Skerbetz, Ryan S Baker, and Anna V Fisher. 2016. Off-task behavior in elementary school children. Learning and Instruction 44 (2016), 128-143.

[27] Karrie E Godwin, Howard J Seltman, Shimin Kai, Ryan S Baker, and Anna V Fisher. [n.d.]. The Variable Relationship Between On-Task Behavior and Learning.

[28] Elizabeth A Gunderson, Gerardo Ramirez, Sian L Beilock, and Susan C Levine. 2012. The relation between spatial skill and early number knowledge: the role of the linear number line. Developmental psychology 48, 5 (2012), 1229.

[29] Mary Hegarty and David A Waller. 2005. Individual differences in spatial abilities. Cambridge University Press.

[30] Sherry Hsi, Marcia C Linn, and John E Bell. 1997. The role of spatial reasoning in engineering and the design of spatial instruction. fournal of engineering education 86, 2 (1997), 151-158.

[31] Yingdan Huang, Mark D Gross, Ellen Yi-Luen Do, and Mike Eisenberg. 2009. Easigami: A reconfigurable folded-sheet TUI. In Proceedings of the 3rd International Conference on Tangible and Embedded Interaction. 107-112.

[32] Nathaniel Hudson, Celena Alcock, and Parmit K Chilana. 2016. Understanding newcomers to 3D printing: Motivations, workflows, and barriers of casual makers. In Proceedings of the 2016 CHI conference on human factors in computing systems. 
384-396.

[33] Nathaniel Hudson, Benjamin Lafreniere, Parmit K Chilana, and Tovi Grossman. 2018. Investigating how online help and learning resources support children's use of 3D design software. In Proceedings of the 2018 CHI Conference on Human Factors in Computing Systems. 1-14.

[34] Yael Kali and Nir Orion. 1996. Spatial abilities of high-school students in the perception of geologic structures. Fournal of Research in Science Teaching: The Official Journal of the National Association for Research in Science Teaching 33, 4 (1996), 369-391.

[35] James Floyd Kelly. 2014. 3D Modeling and Printing with Tinkercad: Create and Print Your Own 3D Models. Que Publishing.

[36] Kimia Kiani, George Cui, Andrea Bunt, Joanna McGrenere, and Parmit K Chilana. 2019. Beyond" One-Size-Fits-All" Understanding the Diversity in How Software Newcomers Discover and Make Use of Help Resources. In Proceedings of the 2019 CHI Conference on Human Factors in Computing Systems. 1-14.

[37] Aytaç Kurtulus and Candas Uygan. 2010. The effects of Google Sketchup based geometry activities and projects on spatial visualization ability of student mathematics teachers. Procedia-Social and Behavioral Sciences 9 (2010), 384-389.

[38] Ghang Lee, Charles M Eastman, Tarang Taunk, and Chun-Heng Ho. 2010. Usability principles and best practices for the user interface design of complex $3 \mathrm{D}$ architectural design and engineering tools. International journal of humancomputer studies 68, 1-2 (2010), 90-104.

[39] Shouming Li, Jules M Marquart, and Craig Zercher. 2000. Conceptual issues and analytic strategies in mixed-method studies of preschool inclusion. fournal of early intervention 23,2 (2000), 116-132.

[40] Marcia C Linn and Anne C Petersen. 1985. Emergence and characterization of sex differences in spatial ability: A meta-analysis. Child development (1985), 1479-1498.

[41] Hod Lipson. 2007. Printable 3D models for customized hands-on education. Mass Customization and Personalization (MCPC) (2007), 6.

[42] Brenna McNally, Leyla Norooz, Alazandra Shorter, and Evan Golub. 2017. Toward Understanding Children's Perspectives on Using 3D Printing Technologies in Their Everyday Lives. In Proceedings of the 2017 Conference on Interaction Design and Children (Stanford, California, USA) (IDC '17). Association for Computing Machinery, New York, NY, USA, 298-303. https://doi.org/10.1145/3078072.3079735

[43] Catarina Mota. 2011. The rise of personal fabrication In: Proceedings of the 8th ACM conference on Creativity and cognition.

[44] Stefanie Mueller. 2017. 3D Printing for Human-Computer Interaction. Interactions 24, 5 (Aug. 2017), 76-79. https://doi.org/10.1145/3125399

[45] Nora S Newcombe and Thomas F Shipley. 2015. Thinking about spatial thinking: New typology, new assessments. In Studying visual and spatial reasoning for design creativity. Springer, 179-192.

[46] Oi-Lam Ng and To Chan. 2019. Learning as Making: Using 3D computer-aided design to enhance the learning of shape and space in STEM-integrated ways. British fournal of Educational Technology 50, 1 (2019), 294-308.

[47] Ann Nishioka and Mike Eisenberg. 1997. Paper Modelling from a Distance: Computational Crafts on the Web. Proceedings of AACE ED-MEDIA/ED-TELECOM 97 (1997), 757-762.

[48] James R Osborn and Alice M Agogino. 1992. An interface for interactive spatial reasoning and visualization. In Proceedings of the SIGCHI conference on Human factors in computing systems. 75-82.

[49] William R Penuel and Brian J Reiser. 2018. Designing NGSS-aligned curriculum materials. Committee to Revise America's Lab Report (2018).

[50] Irene Posch and Geraldine Fitzpatrick. 2012. First steps in the FabLab: experiences engaging children. In Proceedings of the 24th Australian Computer-Human Interaction Conference. 497-500.

[51] Ajay Ramful, Thomas Lowrie, and Tracy Logan. 2017. Measurement of spatial ability: Construction and validation of the spatial reasoning instrument for middle school students. Fournal of Psychoeducational Assessment 35, 7 (2017), 709-727.

[52] Brian J Reiser. 2014. Designing coherent storylines aligned with NGSS for the K-12 classroom. In National Science Education Leadership Association Meeting. Boston, MA.

[53] Andrej Šafhalter, Karin Bakracevic Vukman, and Srečko Glodež. 2016. The effect of 3D-modeling training on students' spatial reasoning relative to gender and grade. Fournal of Educational Computing Research 54, 3 (2016), 395-406.

[54] Chelsea Schelly, Gerald Anzalone, Bas Wijnen, and Joshua M Pearce. 2015. Opensource 3-D printing technologies for education: Bringing additive manufacturing to the classroom. Fournal of Visual Languages \& Computing 28 (2015), 226-237.

[55] Rita Shewbridge, Amy Hurst, and Shaun K Kane. 2014. Everyday making: identifying future uses for 3D printing in the home. In Proceedings of the 2014 conference on Designing interactive systems. 815-824.

[56] Yael Shwartz, Ayelet Weizman, David Fortus, Joe Krajcik, and Brian Reiser. 2008 Middle school science curriculum: Coherence as a design principle. In National Association of Research in Science Teaching.

[57] Rachel Charlotte Smith, Ole Sejer Iversen, and Mikkel Hjorth. 2015. Design thinking for digital fabrication in education. International fournal of ChildComputer Interaction 5 (2015), 20-28.
[58] Abigale Stangl, Jeeeun Kim, and Tom Yeh. 2014. 3D printed tactile picture books for children with visual impairments: a design probe. In Proceedings of the 2014 conference on Interaction design and children. 321-324.

[59] Jeff KT Tang, Tin-Yung Au Duong, Yui-Wang Ng, and Hoi-Kit Luk. 2015. Learning to create 3D models via an augmented reality smartphone interface. In 2015 IEEE International Conference on Teaching, Assessment, and Learning for Engineering (TALE). IEEE, 236-241.

[60] Holly A Taylor and Allyson Hutton. 2013. Think3d!: Training spatial thinking fundamental to STEM education. Cognition and Instruction 31, 4 (2013), 434-455.

[61] David H Uttal and Cheryl A Cohen. 2012. Spatial thinking and STEM education: When, why, and how? In Psychology of learning and motivation. Vol. 57. Elsevier, 147-181.

[62] David H Uttal, David I Miller, and Nora S Newcombe. 2013. Exploring and enhancing spatial thinking: Links to achievement in science, technology, engineering, and mathematics? Current Directions in Psychological Science 22, 5 (2013), 367-373.

[63] Lev Semenovich Vygotsky. 1980. Mind in society: The development of higher psychological processes. Harvard university press.

[64] Jonathan Wai, David Lubinski, and Camilla P Benbow. 2009. Spatial ability for STEM domains: Aligning over 50 years of cumulative psychological knowledge solidifies its importance. fournal of educational Psychology 101, 4 (2009), 817.

[65] Nahathai Wongpakaran, Tinakon Wongpakaran, Danny Wedding, and Kilem L Gwet. 2013. A comparison of Cohen's Kappa and Gwet's AC1 when calculating inter-rater reliability coefficients: a study conducted with personality disorder samples. BMC medical research methodology 13, 1 (2013), 1-7.

[66] Steve Chi-Yin Yuen, Gallayanee Yaoyuneyong, and Erik Johnson. 2011. Augmented reality: An overview and five directions for AR in education. fournal of Educational Technology Development and Exchange (JETDE) 4, 1 (2011), 11. 